Research Article

\title{
Visualization Techniques of Differentially Heated Lid-Driven Square Cavity
}

\author{
Vekamulla Narayana (iD \\ Department of Mathematics, Balaji Institute of Technology \& Science, Narsampet, Warangal-506331, Telangana State, India \\ Correspondence should be addressed to Vekamulla Narayana; vnarayana.wgl@gmail.com
}

Received 10 January 2020; Revised 6 February 2020; Accepted 8 February 2020; Published 9 March 2020

Academic Editor: Irena Lasiecka

Copyright (c) 2020 Vekamulla Narayana. This is an open access article distributed under the Creative Commons Attribution License, which permits unrestricted use, distribution, and reproduction in any medium, provided the original work is properly cited.

In the present study, an attempt is made to explore the flow field inside the differentially heated lid-driven square cavity. The governing equations along with boundary conditions are solved numerically. The simulated results $(100 \leq \operatorname{Re} \leq 1000$ and $0.001 \leq \mathrm{Ri} \leq 10)$ are validated with previous results in the literature. The convection differencing schemes, namely, UPWIND, QUICK, SUPERBEE, and SFCD, are discussed and are used to simulate the flow using the MPI code. It is observed that the computational cost for all the differencing schemes get reduced tremendously when the MPI code is implemented. Plots demonstrate the influences of Re and Ri in terms of the contours of the fluid streamlines, isotherms, energy streamlines, and field synergy principle.

\section{Introduction}

Without external forces such as exterior wind or fans, fluids flow because of density variations. These variations of density inside the enclosures are due to natural and forced convections. There is a general practice in evaluating the vital role of each of the convection type for determining the dominant convection type. Laminar natural and mixed convection in enclosures was broadly analysed both numerically and experimentally with major attention in wide number of engineering and technical applications. Analysis indicates that Richardson number (Ri) characterizes mixed convection flow, where Gr and Re represent the strength of the natural and forced convection flow effects, respectively. The limiting case $\mathrm{Ri} \longrightarrow 0$ and $\mathrm{Ri} \longrightarrow \infty$ correspond to the forced and natural convection flows, respectively. For the past recent decades, lot of experimental and theoretical analysis on laminar free convection in the enclosures is performed.

When $\operatorname{Pr} \geq 1$ and $\operatorname{Pr} \leq 1$, Koseff and Street [1] studied and performed a scale analysis of free and forced convection flow over a vertical wall, and he presented the benchmark results for the transformation from the forced convection dominant flow to natural convection dominant flow and carried out the results which are not the same for fluids. Koseff and Street $[2,3]$ performed a number of experiments in a cavity filled with water and calculated the heat flux at distinct places over the hot floor for the range of Gr and Re and showed that the heat transfer rate is a weak function for the range of $\mathrm{Gr}$ with $2200 \leq \operatorname{Re} \leq 12000$. Mixed convection problem with liddriven flows within an enclosure finds a wide range of applications in various fields of engineering and science such as flow and heat transfer in solar ponds [4], dynamics of lakes [5], thermal-hydraulics of nuclear reactors, and float glass production [6]. The lid-driven cavity problem has been extensively used as a benchmark case for the evaluation of numerical solution algorithms. Moallemi and Jang [7] analysed the effects of Prandtl number (Pr) on a laminar mixed convection heat transfer in a lid-driven cavity. They performed the numerical simulations for two-dimensional laminar flow $(100 \leq \mathrm{Re} \leq 2200)$ and studied the effects of small to moderate Prandtl numbers $(0.01 \leq \operatorname{Pr} \leq 50)$ on the flow and heat transfer characteristics in a square cavity for various values of Richardson number (Ri). The temperature and flow fields in the cavity show the strong influence of Prandtl number, Pr. The local and average Nusselt numbers 
are also reported for various values of $\mathrm{Re}, \mathrm{Ri}$, and Pr. Oztop and Dagtekin [8] studied steady-state two-dimensional mixed convection problem in a vertical two-sided lid-driven differentially heated square cavity. The left and right moving walls were maintained at different constant temperatures while the upper and bottom walls were thermally insulated. Three cases were considered depending on the direction of moving walls and the Richardson number, Ri. They observed that both the Richardson number and the direction of moving walls affect the fluid flow and heat transfer in the cavity. For $\mathrm{Ri} \leq 1$, the influence of moving walls on the heat transfer is the same when the walls move in the opposite direction regardless of which side moves upwards, and the influence is less when both the sides move upwards.

In FVM, the convection term is discretized-based central differencing (CD), which is of a second-order accurate. However, the combination of the explicit time integration and $\mathrm{CD}$ creates unconditionally unstable solutions. In order to attain stability, differencing schemes of a first-order accurate have been introduced. The unsatisfactory behaviour of the first-order schemes with respect to the boundedness and the combined spatial and temporal discretization, introduces an unnecessary dependence of the solution on the time-step used to create it. Hence, it is important to obtain bounded numerical solutions while solving the transport equations. Thus, a good differencing scheme must balance between boundedness and accuracy.

Apart from the issues of accuracy and boundedness, which are essential for accurate calculations, modern differencing schemes are also required to be convergent and computationally inexpensive. The issue of computational cost includes both the additional face-by-face operations required to determine the weighting factors in the total variation diminishing (TVD) and normalised variable diagram (NVD) schemes and the additional effort required to get solutions for steady-state problems. With the development of NVD, the accuracy and boundedness of differencing schemes have been improved at the expense of convergence. For this reason, still there is a need to analyse the convection discretisation schemes. In this paper, numerical examples are given using the high-order convection differencing schemes, namely, Upwind Difference, QUICK, SUPERBEE from a family of TVD schemes, and SFCD from a family of NVD schemes. They are compared with respect to their accuracy and computational time. The SIMPLE algorithm is used to deal with the pressure-velocity coupling. The Gauss-Seidel method of iterative procedure is used to solve the system of algebraic equations. The gird-independent nonuniform mesh is used such that the near wall eddies are captured accurately. The results are validated with those results available in the literature and also shown that the distributed memory parallel processors support greater potential CPU speedup.

To describe flow field and thermal field characteristics from the enormous output data, apart from the traditional plotting tools such as isotherms and streamlines, Hooman [9] introduced and developed a new visualization tool known as the energy flux vectors. These vectors represent the flow of energy and are tangent to heatlines; also the new technique of convection heat transfer called field synergy principle by Guo et al. $[10,11]$ is employed in the present study.

In Section 2, the problem description and the corresponding closed form of the mathematical model are elaborated. The numerical method to solve the equations is outlined. The results are validated with the experimental/ numerical methods available in the literature. Section 3 deals with the results and its discussion. Finally, the conclusions of the present model are listed.

\section{Problem Description and Governing Equations}

The considered square enclosure, along with bottom wall and the half-height of the vertical right and vertical left walls, is differentially heated. For the considered enclosure, the gradient of temperature $\Delta T=T_{H}-T_{C}>0$, and top wall is assumed to be adiabatic as shown in Figure 1.

The conservation of mass, momentum, and energy equations are as follows:

$$
\begin{aligned}
\nabla \cdot \mathbf{V} & =0 \\
(\mathbf{V} \cdot \nabla) \mathbf{V} & =-\nabla P+\frac{1}{\operatorname{Re}} \nabla^{2} \mathbf{V}+\operatorname{Ri} T^{*}, \\
(\mathbf{V} \cdot \nabla) T^{*} & =\frac{1}{\operatorname{Re}} \nabla^{2} T^{*},
\end{aligned}
$$

the equations are subjected to the boundary conditions as $\mathbf{V}=(1,0)$ at $Y=1$ and $\mathbf{V}=0$ at $Y=0$ and also $\mathbf{V}=0$ at $X=0,1$. $T^{*}=1$ when $Y=0(0 \leq X \leq 1)$ and also $T^{*}=1$ when $X=0$, $1(0 \leq Y \leq 0.5)$. Also $T^{*}=0$ when $X=0,1(0.5 \leq Y<1)$ and $\partial T^{*} / \partial Y=0$ at $Y=1$.

In the above equations (1)-(3), $V, T^{*}$, and $P$ denote the field variable's velocity, temperature, and pressures, respectively. The control variables are $\mathrm{Ra}$ and $\mathrm{Pr}$. The above equations are solved by using the open-source software OpenFOAM. The second-order upwind numerical method was used for the calculation of derivatives. The conjugate gradient scheme is used for accelerating the convergence. The convection differencing schemes, namely, UPWIND, QUICK, SUPERBEE, and SFCD, are used to discretize the convection term and are detailed as follows.

In the SUPERBEE method, the field variable $\Phi$ is expressed as a sum of the first-order bounded differencing scheme (UD) and a "limited" higher-order correction (HO) as

$$
\Phi_{f}=(\Phi)_{U D}+\Psi(r)\left[(\Phi)_{H O}-(\Phi)_{U D}\right]
$$

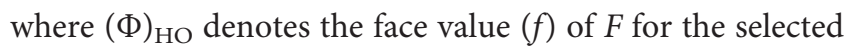
higher-order scheme and $\Psi$ represents the flux limiter function, which is given as

$$
r=\frac{\Phi_{C}-\Phi_{U}}{\Phi_{D}-\Phi_{C}} .
$$

The nodal points $U, C$, and $D$ are selected based on the direction of flow on the face $f$. In this method, the limiter function $\Psi(r)=\max [0, \min (2 r, 1), \min (r, 2)]$. 


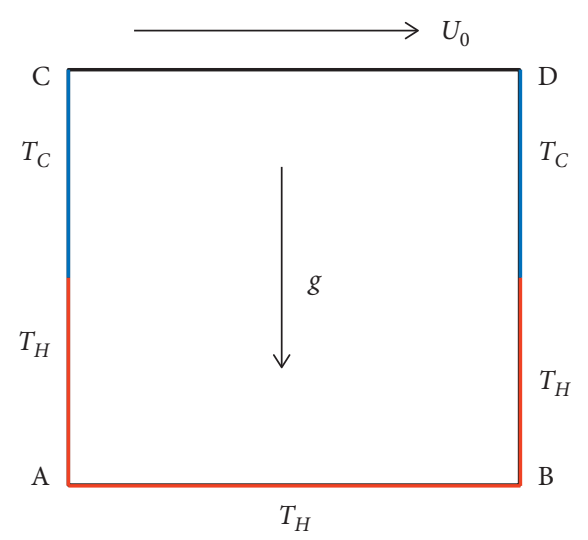

FiguRE 1: Differentially heated lid-driven square cavity.

The self-filtered central differencing (SFCD) scheme employs the boundedness of the upwind differencing scheme (UDS) and the accuracy of the central differencing scheme (CDS). This scheme removes the unphysical extrema whenever they would arise. Basically, this filter is locally shifting towards the UDS in such zones. Thus, in this method, the blending of CDS and UDS will be carried out with a blending factor $\gamma$, ranging from 0 to 1 depending on a computed face $e$, and is defined as

$$
\Phi_{e}^{S F C D}=\gamma_{e} \Phi_{e}^{C D S}+\left(1-\gamma_{e}\right) \Phi_{e}^{U D S} .
$$

The convection differencing schemes, namely, UPWIND, QUICK, SUPERBEE, and SFCD, are compared in terms of $\overline{\mathrm{Nu}}$ using the MPI code and are tabulated in Table 1.

With respect to the CPU time, it is observed that the computational cost for all the differencing schemes get reduced tremendously when the MPI code is implemented. Also, the SFCD scheme gave the $\overline{\mathrm{Nu}}$ results close to those available in the literature.

\section{Results and Discussion}

In this section, the flow is simulated in the $2 \mathrm{D}$ square cavity with a moving lid of velocity $U_{0}$. The simulated results are shown with respect to the streamlines and isotherms and field synergy with respect to the control parameters arising in the system.

Table 2 represents the comparison of the present results with those of Iwatsu et al. [12] and Ouertatani et al. [13] in terms of $\overline{\mathrm{Nu}}$ along the hot wall. It gives good agreement between the present simulated results and the results from the literature. It is also observed that for a fixed $\mathrm{Ri}$, when $\mathrm{Re}$ increases $\overline{\mathrm{Nu}}$ also increases. This shows that conduction starts to prevail for increasing Re.

3.1. Streamlines. When $\mathrm{Ri}$ is small $(<1)$ and Re increases, the primary central vortex appears to play a major role in the fluid flow, and it is changing its location from the top to the bottom due to less buoyancy effects (Figures 2(a)-2(c)). Also as Re increases, two secondary eddies are observed at the bottom part of the cavity because of lid movement. When Ri
TABle 1: Test comparison of convective differencing schemes for the flow in $2 \mathrm{D}$ cavity when $\mathrm{Re}=400$ and $\mathrm{Ri}=1$.

\begin{tabular}{lccc}
\hline Schemes & $\overline{\mathrm{Nu}}$ & $\begin{array}{c}\text { CPU time with MPI } \\
(\mathrm{s})\end{array}$ & $\begin{array}{c}\text { CPU time without MPI } \\
(\mathrm{s})\end{array}$ \\
\hline UPWIND & 0.548 & 8459 & 33836 \\
QUICK & 0.954 & 8157 & 32680 \\
SUPERBEE & 1.235 & 7951 & 31804 \\
SFCD & 1.498 & 5784 & 23136 \\
\hline
\end{tabular}

TABLE 2: Representation of the validation of $\overline{\mathrm{Nu}}$ for different $\mathrm{Re}$ and Ri.

\begin{tabular}{ccccc}
\hline & & & $\mathrm{Ri}$ & \\
& & & & \\
\multirow{4}{*}{100} & Pe & 0.001 & 1 & 10 \\
\hline \multirow{4}{*}{400} & Present work & 1.825 & 1.3187 & 1.085 \\
& Iwatsu et al. [12] & 1.82 & 1.33 & 1.08 \\
& Ouertatani et al. [13] & 1.836 & 1.348 & 1.092 \\
& Present work & 3.88 & 1.498 & 1.168 \\
& Iwatsu et al. [12] & 3.99 & 1.50 & 1.17 \\
& Ouertatani et al. [13] & 3.964 & 1.528 & 1.130 \\
& Present work & 7.11 & 1.839 & 1.388 \\
& Iwatsu et al. [12] & 7.03 & 180 & 1.37 \\
& Ouertatani et al. [13] & 7.284 & 1.856 & 1.143 \\
\hline
\end{tabular}

is increasing from 1 to 10 , it is observed that more convection takes place, which results in the emerging eddy size increasing and forms another secondary vortex (Figures 2(b)-2(i)).

3.2. Isotherms. Figure 3 shows the isotherms in the cavity for different Re and Ri. For small Re and with increase of Ri, the flow starts at the hot bottom wall and forms the horizontal isotherms because of the existence of the conduction mode. When $\mathrm{Re}$ increases with increase of $\mathrm{Ri}$, the enhanced convection also leads to the distorted and asymmetric isotherms, i.e., the isotherms get distorted at the centre of the cavity due to convection. It is also observed that more distortion occurs only in the lower half of the cavity because of the hot wall effects and also high Re. These results clearly show the prevailing convection mode, and they coincide with those shown in Figure 3.

3.3. Energy Streamlines. In the two-dimensional flow, energy streamline is calculated by solving the Poisson equation of the form $\nabla^{2} \Phi=(\nabla \times \bar{E}) \bar{k}$, where $\Phi$ is known as the energy streamfunction and $\bar{k}$ denotes the unit vector. These lines include the energy contribution because of surface forces and energy fluxes. Therefore, the participation of extra energy fluxes from the energy streamfunction is useful for examining the quantitative details. Mallinson and Davis [14] noticed that the energy flux density vectors explore an imaginary energy flow lines, i.e., like path line trace. Mahmud and Fraser [15] revealed the 'free energy streamline' as the streamline of a flow which starts at the wall which is hot, forms rolls that are circular, and later intersects at the wall which is cold. This is because of convective energy participation, and it is negligible in value in comparison with 


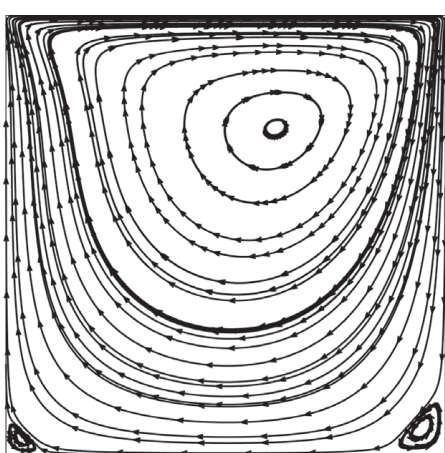

(a)

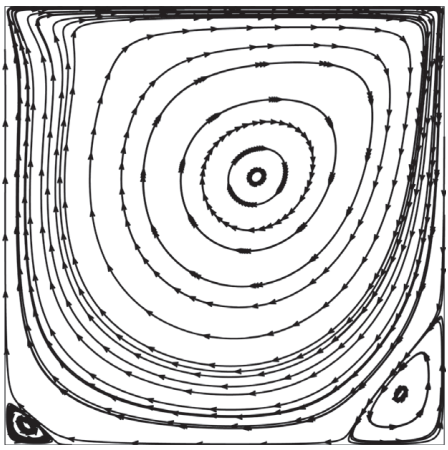

(d)

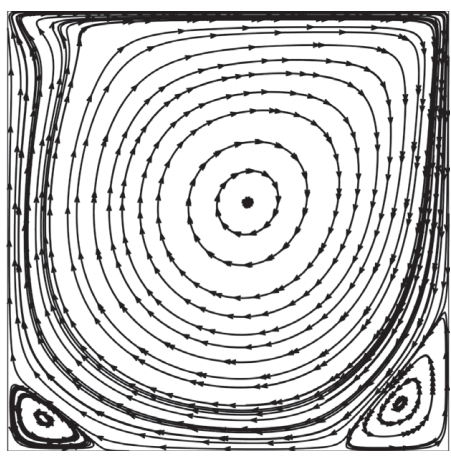

(g)

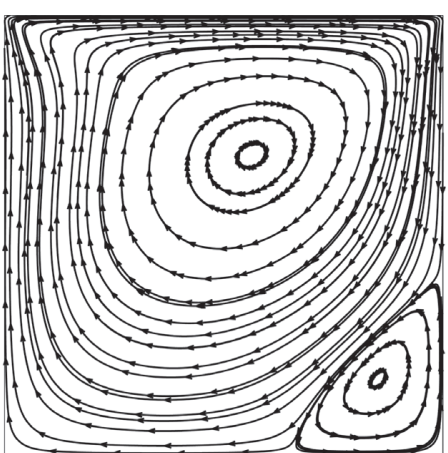

(b)

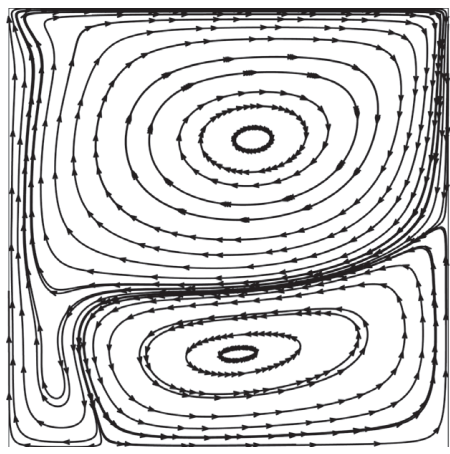

(e)

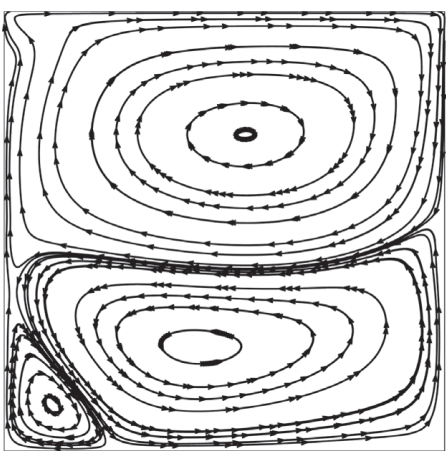

(h)

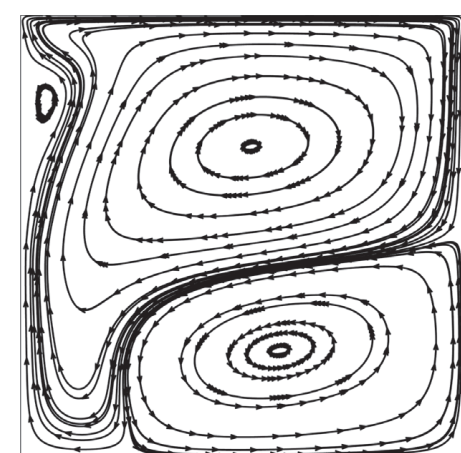

(c)

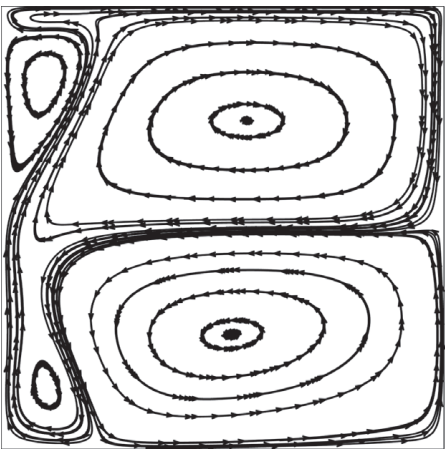

(f)

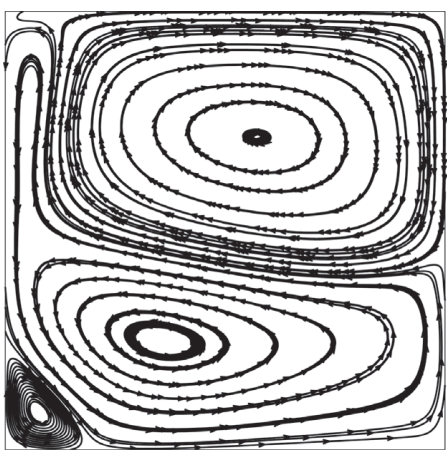

(i)

Figure 2: Simulated streamlines for different Re and Ri values. $(R e, R i)=(a)(100,0.001),(b)(100,1),(c)(100,10),(d)(400,0.001),(e)(400$, $1)$, (f) $(400,10),(g)(1000,0.001),(h)(1000,1)$, and (i) $(1000,10)$.

conductive thermal energy at the locations of modest velocity components. Trapped energy streamline is the streamline that starts at the hot wall, flows through the fluid, and forms a closed circular loop because of kinetic and convective thermal energies dominant over the thermal energy due to conduction. Figure 4 shows the energy streamlines for different Re and Ri. It is observed that free energy streamlines exist only at the boundaries, whereas trapped energy streamlines are formed at the top of the cavity. For small $\mathrm{Ri}$ and with increase of Re, it is clearly observed that the primary vortex location is changing from the top to the bottom and also the size of vortex increases (Figures 4(a), 4(d), 4(g)). Similar effect continues for high $R i$, and also a single vortex breaks into more vortices due to the presence of secondary flows (Figures 4(b), 4(e), 4(h) and Figures 4(c), 4(f), 4(i)).
3.4. Field Synergy. Field synergy principle can disclose the character of improved heat transport (Guo et al. [10, 11]), and it is given by

$$
\beta_{f}=\cos ^{-1}\left(\frac{\mathbf{V} \cdot \nabla T}{|\mathbf{V}||\nabla T|}\right) .
$$

In equation (7), $|\mathbf{V}|,|\nabla T|$, and $\cos \beta_{f}$ represent the scalar fields, while $\mathbf{V}$ and $\nabla T$ represent vector fields. Thus, synergy concludes the strength of the convective heat transfer.

Figure 5 depicts the field synergy angle for different Re and Ri. Blue colour contour indicates that the field synergy angles are small where the velocity vector and temperature gradient are in the same direction. Red colour contour indicates the high synergy angle where the velocity vector and temperature gradient are perpendicular. It is observed that the synergy angle augments as Re and $\mathrm{Ri}$, which leads to the chaotic flow. 


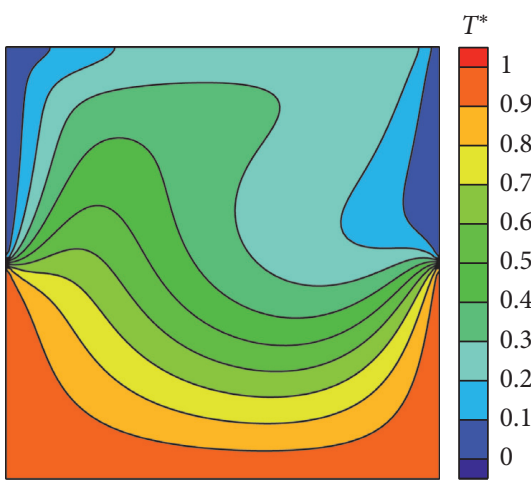

(a)

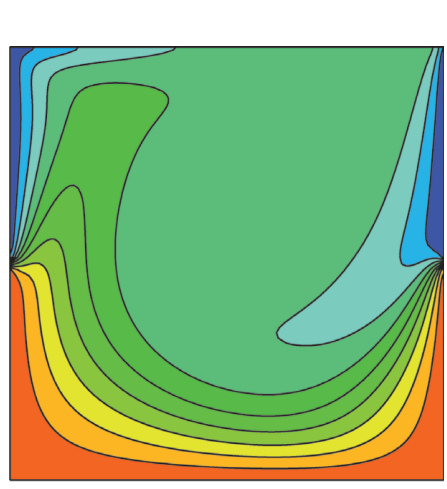

(d)

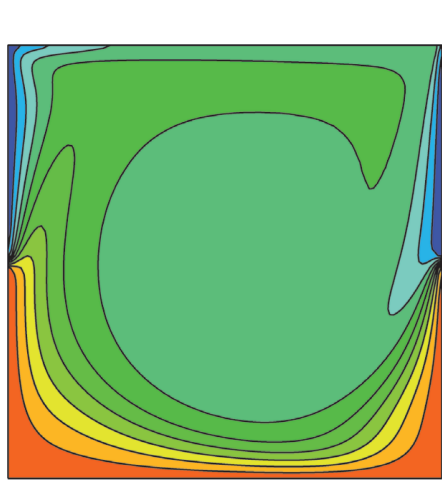

(g)

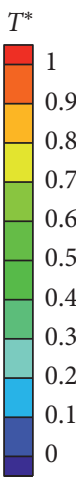

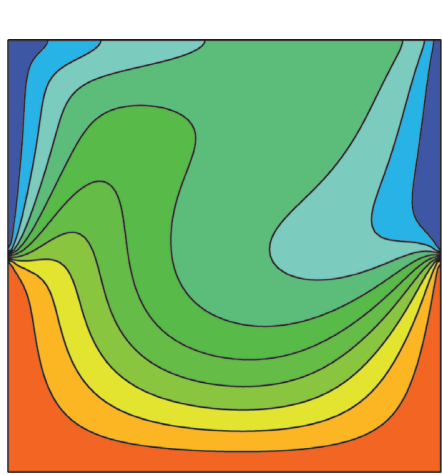

(b)
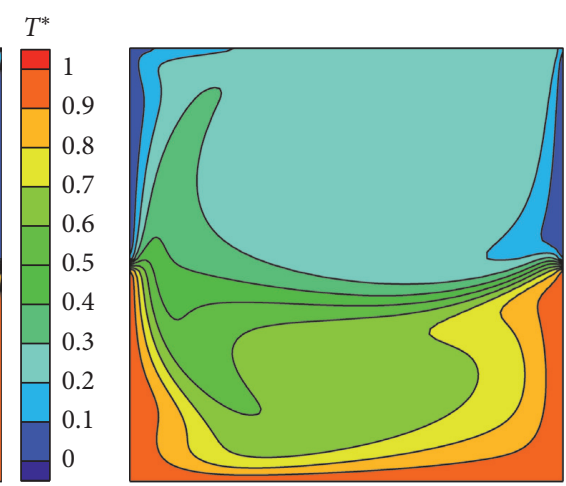

(h)
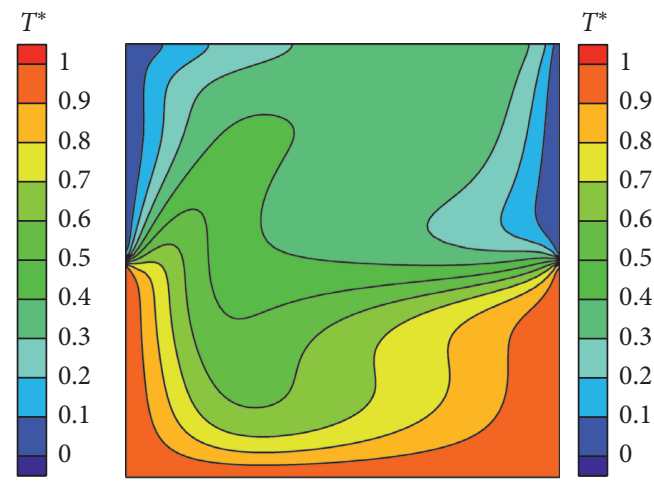

(c)

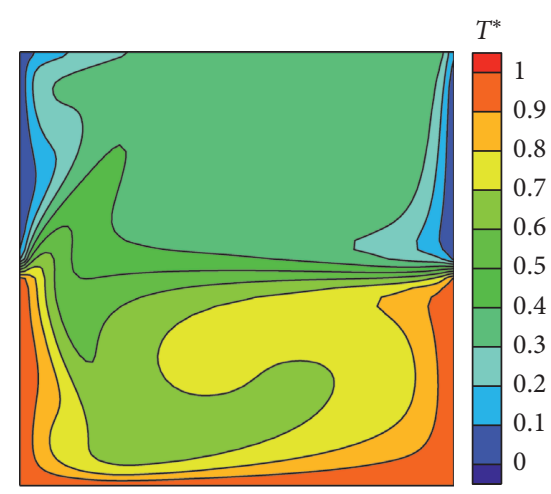

(f)
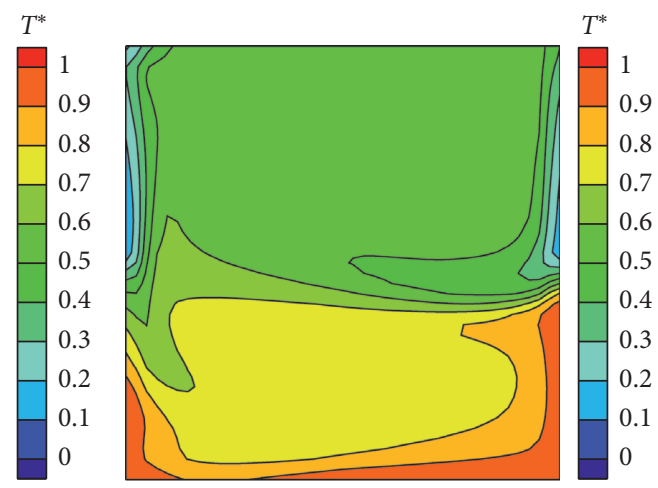

(i)

FiguRe 3: Simulated isotherms for different Re and Ri values. (Re, Ri) = (a) $(100,0.001),(b)(100,1),(c)(100,10)$, (d) $(400,0.001),(e)(400,1)$, (f) $(400,10),(\mathrm{g})(1000,0.001)$, (h) $(1000,1)$, and (i) $(1000,10)$.

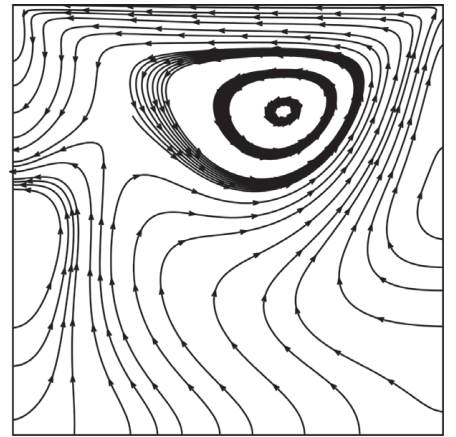

(a)

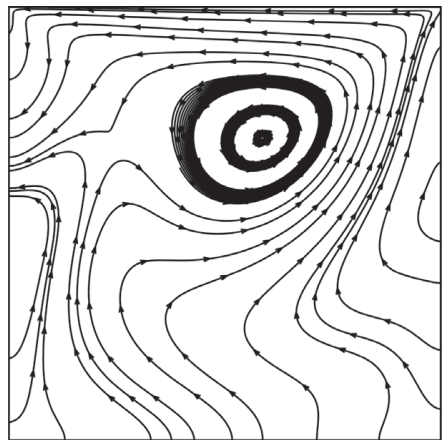

(b)

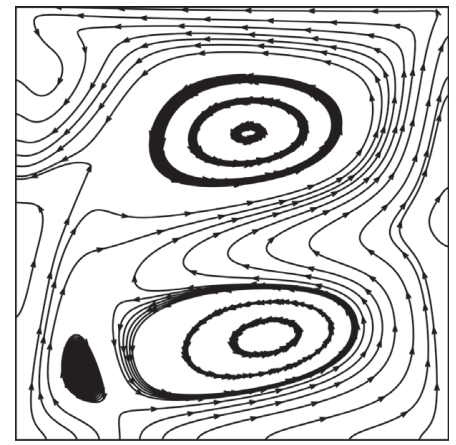

(c)

Figure 4: Continued. 


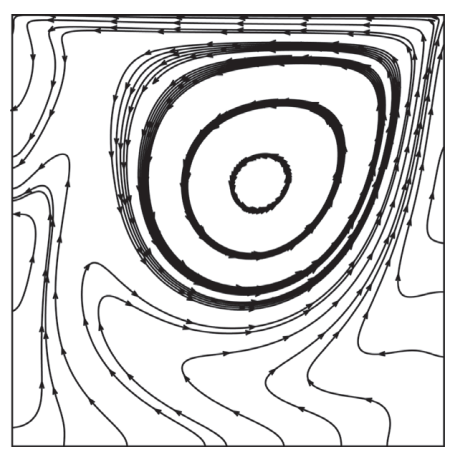

(d)

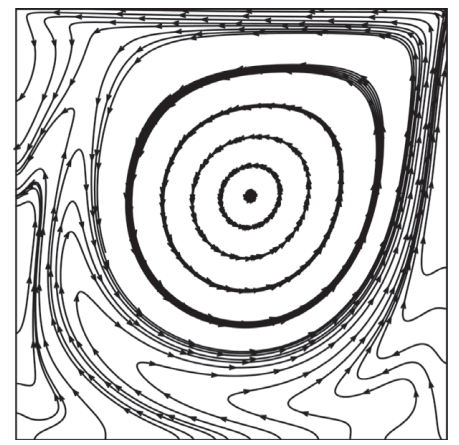

(g)

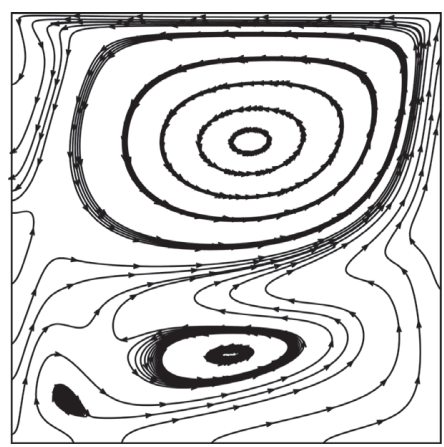

(e)

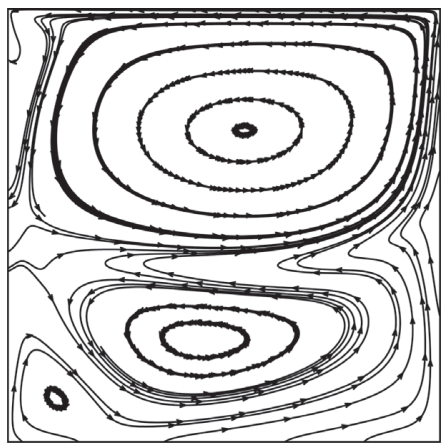

(h)

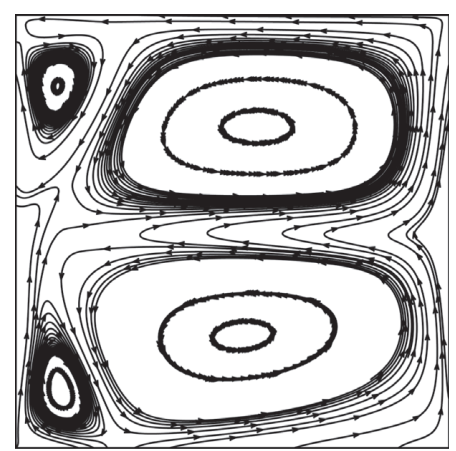

(f)

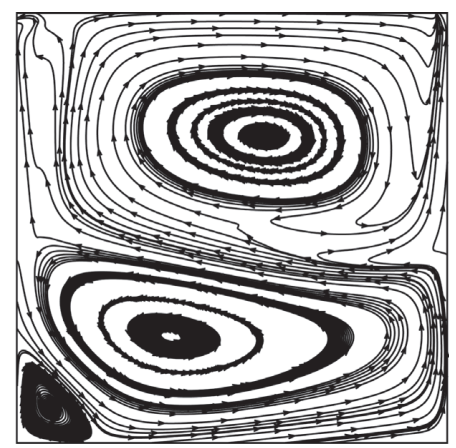

(i)

Figure 4: Simulated energy streamlines for different Re and $R i$ values. $(R e, R i)=(\mathrm{a})(100,0.001),(\mathrm{b})(100,1),(\mathrm{c})(100,10),(\mathrm{d})(400,0.001),(\mathrm{e})$ $(400,1),(f)(400,10),(g)(1000,0.001),(h)(1000,1)$, and (i) $(1000,10)$.

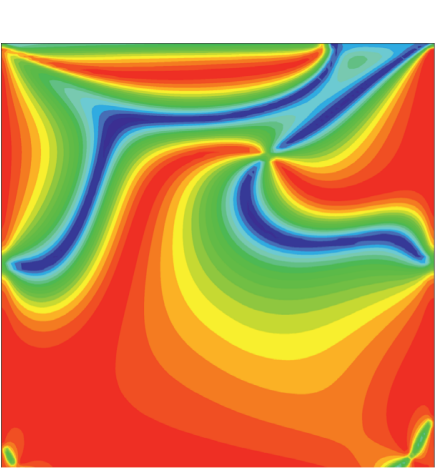

(a)

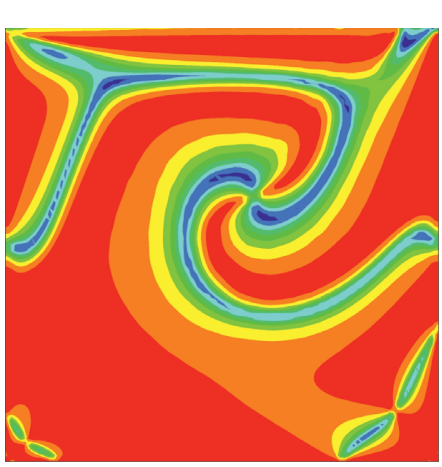

(d)
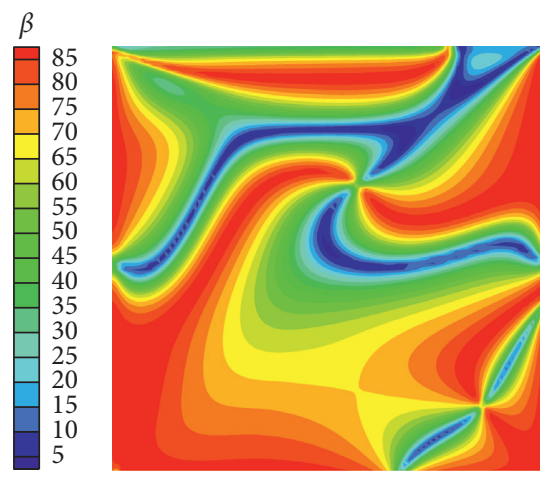

(b)

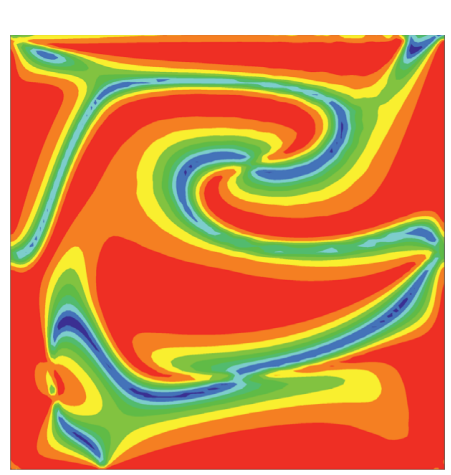

(e)

Figure 5: Continued.
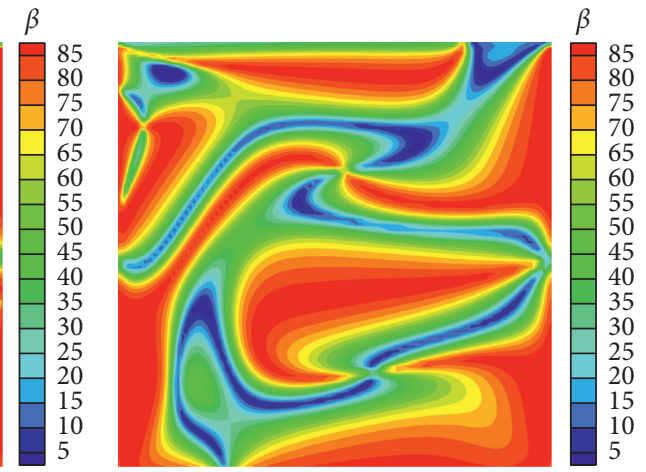

(c)

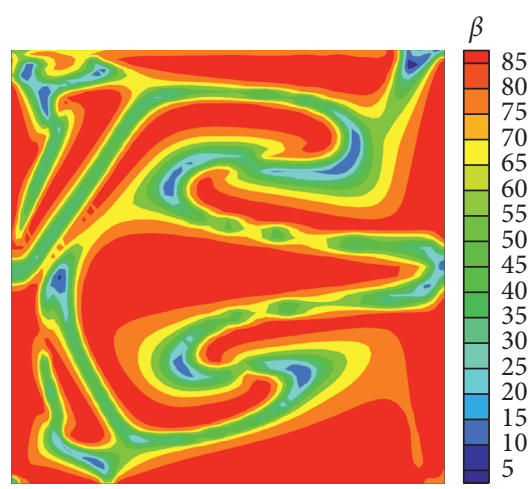

(f) 


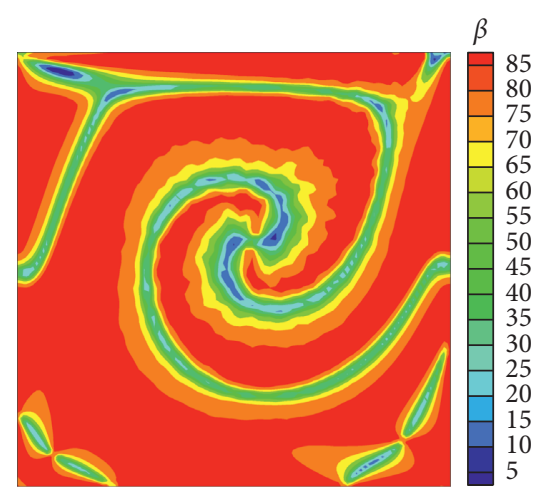

(g)

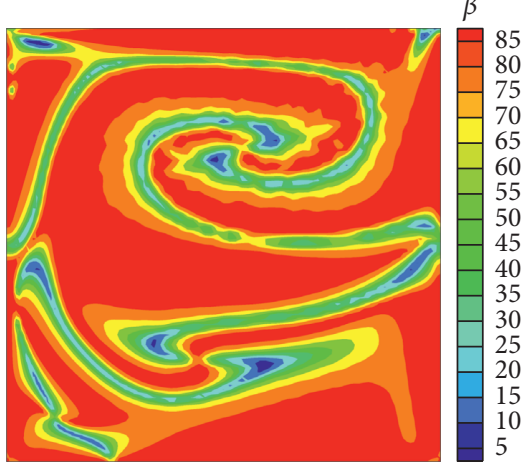

(h)

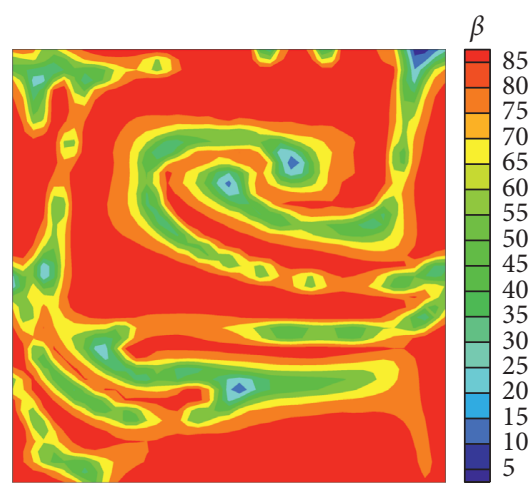

(i)

Figure 5: Simulated field synergy for different Re and Ri values. (Re, Ri) = (a) (100, 0.001), (b) (100, 1), (c) (100, 10), (d) (400, 0.001), (e) (400, $1)$, (f) $(400,10),(g)(1000,0.001),(h)(1000,1)$, and (i) $(1000,10)$.

\section{Conclusions}

The self-filtered central differencing (SFCD) scheme is the combination of the two schemes, namely, the boundedness of the UDS and the accuracy of CDS. It is in fact a central difference scheme with a native filter to remove unphysical extrema whenever they would arise. A parallel computing MPI code is adapted to run the simulations. From the results, it is observed that the NVD scheme gave better results among all the employed convective discretization schemes irrespective of the mesh structure. In this article, SFCD, which is a family of NVD, is used. For a mixed convective differentially heated cavity, when $\mathrm{Ri}$ is small $(<1)$ and as $\mathrm{Re}$ increases, the primary vortex appears to play a major role in the fluid flow, and it is changing its location from the top to the bottom. When $\mathrm{Ri}$ is 10 and 100, the size of emerging eddy is increasing and forms another secondary vortex as Re increases. When $\mathrm{Re}$ is fixed and with increase of $\mathrm{Ri}$, the average Nusselt number also increases.

\section{Data Availability}

No data were used to support this study.

\section{Conflicts of Interest}

The author declares that there are no conflicts of interest.

\section{References}

[1] J. R. Koseff and R. L. Street, "Visualization studies of a shear driven three-dimensional recirculating flow," Journal of Fluids Engineering, vol. 106, no. 1, pp. 21-2, 1984.

[2] J. R. Koseff and R. L. Street, "On end wall effects in a lid-driven cavity flow," Journal of Fluids Engineering, vol. 106, no. 4, pp. 385-389, 1984.

[3] A. Bejan, Convection Heat Transfer, Wiley, New York, NY, USA, 1984, https://onlinelibrary.wiley.com/doi/pdf/10.1002/ 9781118671627.

[4] F. Yi, P. Chen, G. Cai, F. C. M. Lau, S. C. Liew, and G. Han, "Outage-limit-approaching channel coding for future wireless communications: root-protograph low-density parity-check codes," IEEE Vehicular Technology Magazine, vol. 14, no. 2, pp. 85-93, 2019.
[5] P. Chen, Z. Xie, F. Yi, Z. Chen, S. Mumtaz, and J. J. P. C. Rodrigues, "Physical-layer network coding: an efficient technique for wireless communications," IEEE Network, pp. 1-7, 2020.

[6] F. Yi, G. Han, P. Chen, F. C. M. Lau, G. Chen, and L. Wang, "A survey on DCSK-based communication systems and their application to UWB scenarios," IEEE Communications Surveys \& Tutorials, vol. 18, no. 3, 2016.

[7] M. K. Moallemi and K. S. Jang, "Prandtl number effects on laminar mixed convection heat transfer in a lid-driven cavity," International Journal of Heat and Mass Transfer, vol. 35, no. 8, pp. 1881-1892, 1992.

[8] H. F. Oztop and I. Dagtekin, "Mixed convection in two-sided lid-driven differentially heated square cavity," International Journal of Heat and Mass Transfer, vol. 47, no. 8-9, pp. 1761-1769, 2004.

[9] K. Hooman, "Energy flux vectors as a new tool for convection visualization," International Journal of Numerical Methods for Heat \& Fluid Flow, vol. 20, no. 2, pp. 240-249, 2010.

[10] Z. Y. Guo, D. Y. Li, and B. X. Wang, "A novel concept for convective heat transfer enhancement," International Journal of Heat and Mass Transfer, vol. 41, no. 14, pp. 2221-2225, 1998.

[11] Z. Y. Guo, W. Q. Tao, and R. K. Shah, "The field synergy (coordination) principle and its applications in enhancing single phase convective heat transfer," International Journal of Heat and Mass Transfer, vol. 48, no. 9, pp. 1797-1807, 2005.

[12] R. Iwatsu, J. M. Hyun, and K. Kuwahara, "Mixed convection in a driven cavity with a stable vertical temperature gradient," International Journal of Heat and Mass Transfer, vol. 36, no. 6, pp. 1601-1608, 1993.

[13] N. Ouertatani, N. B. Cheikh, B. B. Beya, T. Lili, and A. Campo, "Mixed convection in a double lid-driven cubic cavity," International Journal of Thermal Sciences, vol. 48, no. 7, pp. 1265-1272, 2009.

[14] G. D. Mallinson and G. D. V. Davis, "Three-dimensional natural convection in a box: a numerical study," Journal of Fluid Mechanics, vol. 83, no. 1, pp. 1-31, 1977.

[15] S. Mahmud and R. A. Fraser, "Visualizing energy flows through energy streamlines and pathlines," International Journal of Heat and Mass Transfer, vol. 50, no. 19-20, pp. 3990-4002, 2007. 